Reprod. Nutr. Dévelop., 1986, 26 (1 B), 291-292.

\title{
Dégradation des galactolipides par les protozoaires et les bactéries du contenu du caecum de cheval
}

Annie BONHOMME

\author{
L.A. C.N.R.S. $n^{\circ} 138$, Groupe de Zoologie et Protistologie, \\ Les Cézeaux, B.P. 45, 63170 Aubière \\ et Laboratoire de Zoologie, U.E.R. Sciences, \\ B.P. 347, 51062 Reims Cedex.
}

Summary. Leaf lipids have a high content of galactolipids localized in chloroplasts. Cellfree extracts of caecum bacteria and protozoa (which ingest chloroplasts) hydrolyzed mono and digalactosyldiglycerides, and possessed both $\alpha$ and $\beta$ galactosidase activities.

Dans le caecum de cheval, les Protozoaires s'attachent au substrat végétal et ingèrent des chloroplastes dont ils dégradent la structure (Bonhomme, 1985) et les constituants. Les galactosyldiglycérides, présents dans les thylacoïdes des chloroplastes contiennent des acides gras polyinsaturés en $C_{18}$. Protozoaires et bactéries saturent les doubles liaisons de l'acide linolénique (Bonhomme, 1983). Le but de ce présent travail est d'étudier la dégradation des galactosyldiglycérides par ces microorganismes.

\section{Matériel et méthodes.}

Obtention des extraits enzymatiques. - Le contenu de caecum de cheval fraîchement abattu est filtré sur gaze puis centrifugé à $300 \mathrm{~g}(5 \mathrm{~min})$. Le culot (PB) comporte protozoaires et bactéries. Par centrifugation du surnageant à $27000 \mathrm{~g}$ (30 min), on obtient un culot comportant des bactéries (B) et un surnageant qui, filtré sur millipores $(0,22 \mu \mathrm{m})$ constitue la fraction liquide sans microorganismes (LC). Les culots PB et B sont lavés, centrifugés, soumis aux ultrasons, centrifugés à $33000 \mathrm{~g}(20 \mathrm{~min})$; les surnageants filtrés sur millipores $(0,22 \mu \mathrm{m})$ constituent les extraits enzymatiques PB et B. Plusieurs extractions enzymatiques ont été réalisées sur différents contenus de caecum. Trois fractions PB comportent le genre Cycloposthium (PB Cycl), deux fractions PB comportent le genre Blepharocorys (PB BI).

Hydrolyse par les extraits d'un di-galactosyldiglycéride (DGDG) et d'un monogalactosyldiglycéride (MGDG). - $1 \mathrm{ml}$ d'extrait enzymatique est mis en incubation in vitro dans une solution de $\mathrm{NaHCO}_{3} 5,8 \mathrm{mM}(1 \mathrm{ml})$ en présence de $500 \mu \mathrm{g}$ de DGDG et de MGDG pendant $7 \mathrm{~h}$ et $23 \mathrm{~h}$ à $38^{\circ} \mathrm{C}$. Le galactose libéré est dosé selon la méthode microcuprimétrique (Nelson, 1944) (absorption à $520 \mathrm{~nm}$ ).

Activités $\alpha$ - et $\beta$-galactosidasiques des extraits. $-0,6 \mathrm{ml}$ d'extrait enzymatique est mis en incubation in vitro pendant $2 \mathrm{~h}$ à $38^{\circ} \mathrm{C}$ dans une solution tampon phosphate $0,1 \mathrm{M}(\mathrm{pH} 6,5)(0,6 \mathrm{ml})$ avec $0,2 \mathrm{ml}$ d'une solution de $\mathrm{p}$. nitrophényl- $\alpha$-Dgalactoside $(3 \mathrm{mg} / \mathrm{ml})$ d'une part et dans une solution tampon citrate $0,1 \mathrm{M}(\mathrm{pH}$ $4,6)(0,6 \mathrm{ml})$ avec $0,2 \mathrm{ml}$ d'une solution de $\mathrm{p}$. nitrophényl- $\beta$-D-galactoside ( $3 \mathrm{mg} / \mathrm{ml}$ ) d'autre part. La réaction est stoppée par addition de $2 \mathrm{ml}$ de tampon borate $0,2 \mathrm{M}$ de $\mathrm{pH} 9,8$. L'activité enzymatique est estimée par la libération de paranitrophénol (absorption à $405 \mathrm{~nm}$ ). 
Résultats et discussion. La fraction B hydrolyse les mono- et les digalactosyldiglycérides ; cependant la libération du galactose du MGDG est obtenue après un temps d'incubation relativement long (tabl. 1). La fraction PB étant composée essentiellement de protozoaires, les lavages ne peuvent être répétés sans risque de perdre toute activité enzymatique. Avec DGDG l'hydrolyse par la fraction PB est environ 6 fois supérieure à celle réalisée par la fraction $B$ (tabl. 1). Les deux genres de Protozoaires ciliés des fractions PB étudiées possèdent des activités $\alpha$-et $\beta$-galactosidasiques (tabl. 2). L'activité $\alpha$-galactosidasique se révèle plus importante. La fraction sans microorganismes (LC) ayant une activité négligeable, les $\alpha$ - et $\beta$-galactosidases sont donc liées aux cellules bactérienne et protozoale. Contrairement au genre Epidinium sp. du rumen (Bailey et Howard, 1963), Cycloposthium et Blepharocorys ont une activité $\beta$-galactosidasique et lipolytique (Bonhomme, 1976). L'action des galactosidases s'accompagne donc d'une lipolyse. L'hydrogénation des acides gras insaturés ne peut alors se faire que lorsque ceux-ci sont libérés de la liaison ester.

TABL. 1. - Hydrolyse d'un di-galactosy/diglycéride et d'un mono-galactosyldig/ycéride par les extraits enzymatiques des fractions $\angle C, B$ et $P B$ du contenu du caecum.

\begin{tabular}{|c|c|c|c|c|c|}
\hline \multirow{3}{*}{\multicolumn{2}{|c|}{ Extraits enzymatiques }} & \multicolumn{4}{|c|}{$\mu \mathrm{M}$ de galactose $/ \mathrm{ml} / \mathrm{h} / \mathrm{mg}$ de protéine } \\
\hline & & \multicolumn{2}{|c|}{ DGDG } & \multicolumn{2}{|c|}{ MGDG } \\
\hline & & $7 \mathrm{~h}$. & $23 \mathrm{~h}$. & $7 \mathrm{~h}$. & $23 \mathrm{~h}$. \\
\hline $\begin{array}{l}\text { Liquide acellulaire } \\
\text { Bactéries } \\
\text { Protozoaires + Bactéries }\end{array}$ & $\begin{array}{l}\text { LC } \\
\text { B } \\
\text { PB Cycl(1) } \\
\text { PB Cycl(2) } \\
\text { PB BI }\end{array}$ & $\begin{array}{r}2,6 \\
7,6 \\
48\end{array}$ & $\begin{array}{r}2,4 \\
8,8 \\
52\end{array}$ & $\begin{array}{c}/ \\
/ \\
26,4 \\
18,6\end{array}$ & $\begin{array}{r}4,4 \\
24,9 \\
33,5 \\
19,3\end{array}$ \\
\hline
\end{tabular}

Valeur protéique des extraits enzymatiques : LC : $1,2 \mathrm{mg} / \mathrm{ml}$ d'albumine bovine ; $B: 1,6 \mathrm{mg} / \mathrm{ml} ; \mathrm{PB}$ $\operatorname{cycl}(1): 1,3 \mathrm{mg} / \mathrm{ml}$; $\mathrm{PB} \operatorname{cycl}(2): 0,7 \mathrm{mg} / \mathrm{ml} ; \mathrm{PB} \mathrm{Bl}: 0,8 \mathrm{mg} / \mathrm{ml}$.

TABL. 2. - Activités $\alpha$-et $\beta$-galactosidasiques des extraits enzymatiques des fractions $\angle C, B$ et $P B$ sur le nitrophényl- $\alpha$-galactoside et sur le nitrophényl- $\beta$-galactoside.

\begin{tabular}{lccc} 
& & & $\mu \mathrm{M}$ nitrophényl/h/mg de protéine \\
\cline { 3 - 4 } & Extraits enzymatiques & $\mathrm{NP} \alpha \mathrm{G}$ & $\mathrm{NP} \beta \mathrm{G}$ \\
\hline Liquide acellulaire & $\mathrm{LC}$ & 5,7 & 4,3 \\
Bactéries & $\mathrm{B}$ & 153 & 47,2 \\
Protozoaires + Bactéries & $\mathrm{PB} \mathrm{Cycl}$ & 320 & 69 \\
& PB BI & 249 & 25,5
\end{tabular}

Valeur protéique des extraits enzymatiques LC : $1,2 \mathrm{mg} / \mathrm{ml}$ d'albumine bovine ; B : $1,3 \mathrm{mg} / \mathrm{ml}$; $\mathrm{PB}$ cycl : $0,6 \mathrm{mg} / \mathrm{ml}$; $\mathrm{PB} \mathrm{Bl}: 0,7 \mathrm{mg} / \mathrm{ml}$.

Bailey R. W., Howard B. H., 1963. Carbohydrases of the rumen ciliate Epidinium caudatum. Biochem. J., 87, 146-151.

Bonhomme A., 1976. Activité lipolytique des ciliés et des bactéries du caecum des Equidés. C. R. Acad. Sci. Paris, 282, 1605-1608.

Bonhomme A., 1983. Biohydrogénation des acides gras insaturés des chloroplastes par les protozoaires et les bactéries du contenu du caecum de cheval. Arch. Protistenk, 127, 399-404.

Bonhomme A., 1985. Attachement des Ciliés du caecum de cheval aux fragments végétaux. Dégradation des chloroplastes. Attachement des bactéries aux ciliés du caecum. Reprod. Nutr. Dévelop., 25, 127-139.

Nelson N., 1944. A photometric adaptation of the Somogyi method for the determination of glucose. J. biol. Chem., 153, 375-380. 